\title{
A Multidimensional Fuzzy Knowledge-based System for Optimizing Wireless Local Area Networks Performance
}

\author{
Imeh J. Umoren \\ Department of Computer Science \\ Akwa Ibom State University (AKSU) \\ Mkpat Enin, Akwa Ibom State, Nigeria
}

\author{
Samuel B. Okon \\ Department of Computer Science \\ National Open University of Nigeria (NOUN) \\ FCT, Abuja, Nigeria
}

\begin{abstract}
With the dawn of Wireless Local Area Networks (WLAN), network operators of third generation (3G) and fourth generation (4G) networks can properly address traffic requirements through subscribers and hotspot locations. Primarily, a significant aspect to consider is the issue of performance leading to Quality of Service (QoS) of mobile data networks allow subscribers to experience seamless and ubiquitous services as well as very high data rates. In this paper, we study the existing problem of network degradation which impact the provision of such seamless connectivity. In network performance, most indicators for performance optimization, includes Packet loss, Packet delay and Jitter (PLPDJ. As wireless networks evolve, demand for information services with high reliability, quick response times (QRT) and ubiquitous connectivity continues to upsurge rapidly. These issues are commonly affected by wireless networks inherent variances from wireline networks. Hence, network traffic metrics; latency, packet Loss and packet delay in certain wireless environments experienced some challenges in networks performance. To overcome these challenges, we consider network performance optimization techniques and proposed a framework using Type 1 Fuzzy knowledge-based approach for efficient WLAN performance. First, a performance measures on a typical wireless local area network with IP address 102.89.2.166 was carried out for a period of twenty-one (21) days based on specified performance metrics. Results shows that the average latency on a given WLAN was $11399 \mathrm{~ms}(\mathbf{0 . 1 9 m})$ as compared to jitter which was $1076 \mathrm{~ms}$ $(\mathbf{0 . 0 1 7 m})$. Again, the download speed was established at 191.46Mbs compared to Upload speed which was at 35.7Mbps. Secondly, we obtain statistical operational field data and carried out simulation with the proposed model. Results indicates a minimization on congestions on the representative network environment which shows efficient network performance. Consequently, the evaluation carried out with Triangular Membership Functions (TMF) demonstrates an optimized WLAN Performance with QOS provisioning.
\end{abstract}

\section{Keywords}

Quick Response Time (QRT), Quality of Service (QoS), Packet loss, Packet delay and Jitter

\section{INTRODUCTION}

Over the past decades, there has been a rapid growth in the deployment and use of wireless networks. The advancement in technology is influenced by the availability and use of wireless networks. Most of our devices make use of wireless network and it is of great important that the performance of the network be seriously considered. A good Network Performance helps in facilitating movement and communicating especially in our business and other sectors. The current-generation of wireless systems can provide users with high-speed data services at rates substantially higher than those of the previous generation. As a result, the demand for information services with high reliability, fast response times, and ubiquitous connectivity continues to upsurge rapidly and open the space for performance and optimization. However, optimizing wireless performance networks incorporate a broad range of technologies, including, electronic devices, antennas, digital signal processing algorithms, network control protocols, and cryptography. With this, Application of computational intelligence technique in network, operation, and optimization aims at improving QoS in a wireless network. The increase in the number of mobile users and the overwhelming demand of offered services by the network providers affect the network QoS and a high demand for optimization emerges.

\section{RELATED LITERATURE}

A number of studies have analyzed the performance of wireless networks. We summarize a representative sample of the existing work below.

[9] proposed Network Performance Optimization: A Case Study of Enterprise Network Simulated in OPNET. An enterprise network of an educational institute was considered to optimize the performance. The complete network infrastructure which is comprised of approximately 1200 nodes spread across 5 buildings, was simulated in OPNET first, and after applying appropriate solutions for the flaws that have been highlighted, their proposed system only focuses optimizing network within a building and as such was not open to some consideration as congestions which to a large extent affect network performance.

[5] present a report on Evaluation of WLAN Security and Performance, the report considered the reality of Wireless LAN security and performance. It also provide an overview of security mechanisms and explains how security works in Wireless LAN networks. An in-depth analysis of the Wired Equivalent Privacy (WEP), Wi-Fi protected access (WPA) and WPA2 standards was presented. The security vulnerabilities that exist in these services were analyzed and explained. Experiment involving four devices (Dlink, Linksys, nanostationloco2 and a WBS) was carried out. It was discovered from the literature that most people prefer using WEP though least secure. The study further proved that WEP can be compromised with $100 \%$ success. Again, it was also discovered during the experiment that factors such as line of sight, obstruction, distance, antenna type, channel being used grossly affect network performance.

[17] proposed a Cross-layer schemes for performance optimization in wireless networks to address how to minimize end-to-end delay through joint optimization of network layer routing and link layer scheduling. Two cross-layer schemes, a loosely coupled cross-layer scheme and a tightly coupled crosslayer scheme, were proposed. The two cross-layer schemes 
involve interference modeling in multihop wireless networks with omnidirectional antenna. Based on the interference model, multicast schedules were optimized to minimize the total end-to-end delay. The study also addressed how to leverage the spatial multiplexing function of MIMO links to improve wireless network throughput. The interference model, routing, spatial multiplexing, and scheduling were jointly considered in one optimization model. Their study only focuses on how to minimize the end-to-end delay and how to maximize the throughput in wireless networks, but did not put into considerations other factors that affects network performance such as congestions.

[5] study Network Performance Analysis Within A Local Area network, in their research work, network performance of a local area network within a wireless and wired network was analyzed. It was observed that wired networks constraints like mobility and expensive cabling. But wireless communication is a flexible data communication system implemented as extension to an alternative to wired communication. The bandwidth and the services provided by the wireless communication networks are similar to that provided by the wired networks. Computing the viability and performance of computer networks in real time can be very expensive task. In the study, performance of wireless and wired networks as well as comparison was evaluated using OPNET (Optimized Engineering Tools) simulation tool. for wired network, collision count, traffic received, delay and throughput. In wireless network, data dropped, traffic received, media access delay and throughput was investigated for comparison. Hence, both wired and wireless networks performance parameters was established.

[10] Proposed Optimization of Wireless Network Security System, the purpose of their study was to review contemporary wireless network protocols and areas that affect the ability of wireless fidelity (Wi-Fi) technology to secure data transmitted over wireless networks. Their research approach takes the form of a case study, in collating the methods used by existing protocols in the implementation of wireless Security Trust Models within their networks Wireless protected access Wi-Fi protected access; version 2 (WPA2) protocol has provided a more secure means for securing wireless networks but has only provided stronger encryption as it has a longer key which takes longer to decipher, their proposed study only focuses on the network security aspect.

[12] Richa Rai et al, (2016) in their study Performance Analysis of Fuzzy based RED for Congestion Control in MANET, employ an active queue management (AQM) scheme i.e. random early detection (RED) AQM algorithm which was modified using fuzzy logic MATLAB tool, in such a manner that the efficient and reliable performance of the network can be maintained and improved as well. The results were verified with the help of QualNet 6.1 network simulator, but their proposed scheme did not consider the major key performance parameter in network performance.

[1] proposed a Computer Network Performance management using a Simple Network Management Protocol, their research study the performance management for network and how to measure performance metrics. Performance management refers to the methodology of understanding the behavior of network and its elements in response to traffic demands, measuring and reporting on the network to maintain the network performance at an acceptable level. In their research SNMP protocol was used for network performance evaluation with a given polling time considering all tradeoffs such as Class of service Delay/Utilization. A Wide range of network device parameters like system uptime, interface status, interface packet statistics, routing protocol statistics etc. are treated as different objects by the SNMP. These objects are maintained by the server portion of SNMP software, known as SNMP agent, running on network devices like routers and switches. SNMP client program known as the SNMP manager, runs on a remote machine and queries these objects, through SNMP Get requests, to know the status of the network device.

[4] proposed Performance Optimization Model in 802.11n Networks Using Multi-Objective Programming, their propose system aims to develop a method for finding an alternative to analyze the performance of wireless networks (for this case $802.11 \mathrm{n})$ using Multi-objective Programming. Their formulated model allows determination of optimal values (vector of feasible solution) for a network with infrastructure 802.11n (access points with this technology) that determine the maximum capacity that could have the links depending the technology. However, their limitation was that the solution that shows up is a local optimum that means it is possibly in a certain moment there could be another solution type that equally maximizes the performance of the network.

The work of [15] demonstrated that, congestion, packet loss and delay have strong influence on the performance of WSNs. In order to implement a realistic sensor network policy to resolve the problem of data delay and avoidance of collisions that lead to packet losses, their work develop a system that guarantees QoS in WSNs using Fuzzy Logic Controller (FLC) for sensitivity analysis of the effect of adaptive forward error correction (AFEC). [14] proposed a Handover Manageability and Performance Model for Mobile Communication Networks then formulated a model for soft handoff in CDMA networks by initiating an overlap region between adjacent cells which facilitating the derivation of handoff manageability performance model. The paper employed an empirical modeling approach to support their analytical findings, measure and investigated the performance characteristics of a typical communication network over a specific period in an established cellular communication network operator.

[16] considered a Fuzzy Knowledge-Based (FKB) approach with Triangular Membership Function (TMF) for evaluation of input parameters on selected three (3) mobile network operators in Niger Delta region and recordings were made ver periods of 21 days to ascertain service capacity. The model for optimizing mobile broadband networks was based on the test data. Results demonstrate that, the selected network operators vary in Quality of Service (QoS). Comparison in terms of signal strength, packet loss and data rate were observed at the instance of six (6) scenarios, Operator x provides reasonable Data rate of about $51.93 \mathrm{mbps}$ (lowest download speed), Operator y performed efficiently on packet loss with about $0.01 \%$ loss of packet and Operator $\mathrm{z}$ performed excellently well on signal strength of $98.23 \%$ for networks QoS and user Quality of Experience (uQoE) provisioning

Nevertheless, this research work adopts Fuzzy Based Approach in order to improve upon the existing system, to optimized network performance in Next generation wireless network taking in cognizance of the key performance metrices of network performance. This approach is intending to provide efficiency in optimization. New parameter is introduced into the optimization system such as packet loss, jitter and packet delay which is considered for the implementation to provide basis for effective performance of the network.

\section{PROPOSED SYSTEM DESIGN}

A system is group of interacting, interrelated, or interdependent 
elements forming a complex whole. The systems design is the overall plan or model that shows how the system will meet its information requirements. Essential activities in system design encompasses developing system-level technical requirements and top-level system designs evaluating the design's capability to meet the system requirement.

\subsection{Proposed System Architecture}

Architecture is the essential organization of a system personified in its components, their relationship to each other and the environment, and its principles governing its design and development. Figure 1 Shows Architecture of the Proposed System.

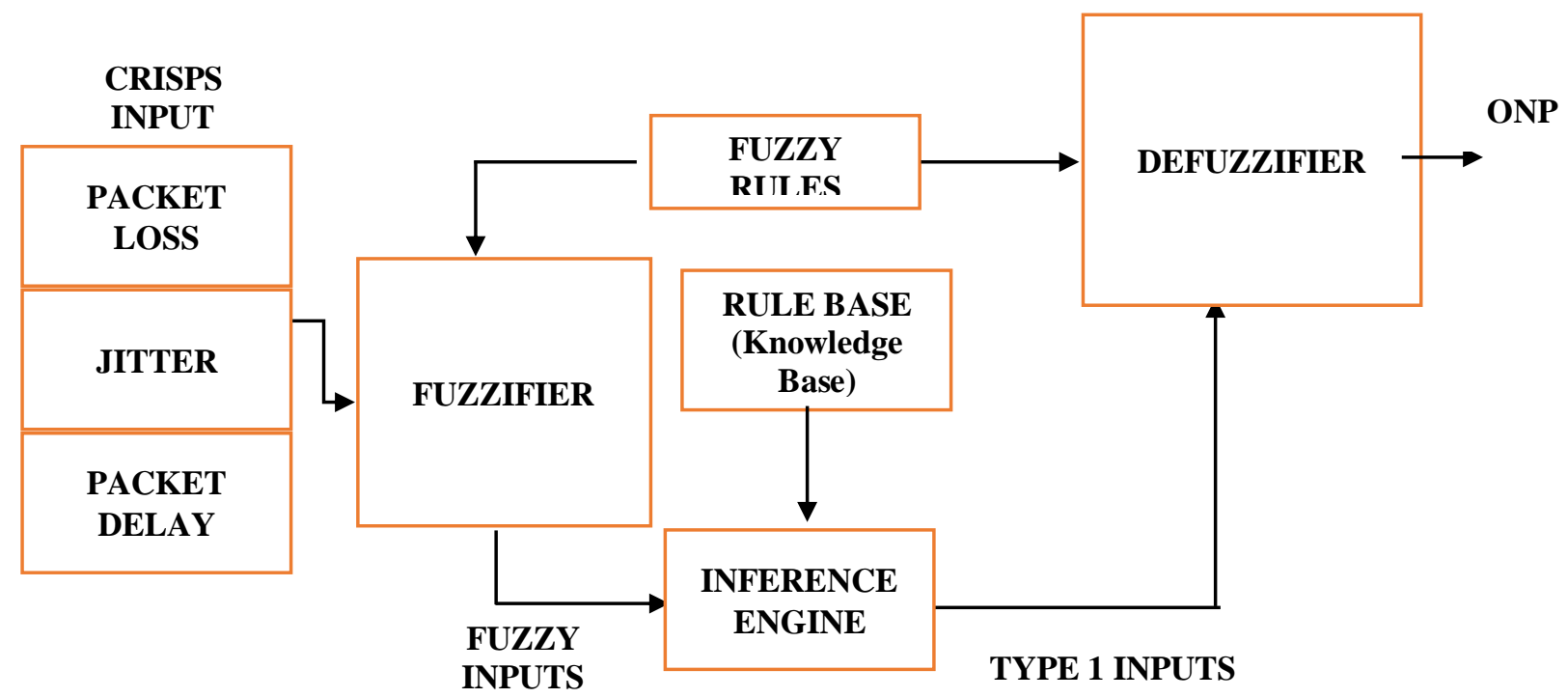

\section{SYSTEM DESIGN}

A system is a set of interrelating parts, created for some particular purpose. Essential activities in system design include developing system-level technical requirements and top-level system designs and assessing the design's ability to meet the system requirements. The system design involves setting of system specification to be adhered to during system implementation.

\subsection{Data Collection}

Data collection is a procedure of collecting, measuring and analyzing accurate insights for research using standard validated techniques. A researcher can evaluate their hypothesis on the basis of collected data. In most cases, data collection is the primary and most important step for research, irrespective of the field of research. The approach of data collection is different for different fields of study, depending on the required information. The most critical objective of data collection is ensuring that information-rich and reliable data is collected for statistical analysis so that data-driven decisions can be made for research. In this research, Network

Table 1: Network Performance dataset

\begin{tabular}{|l|l|l|l|l|l|l|}
\hline Date & IP Address & $\begin{array}{l}\text { Download } \\
\text { Speed }\end{array}$ & $\begin{array}{l}\text { Upload } \\
\text { Speed }\end{array}$ & $\begin{array}{l}\text { latency } \\
\text { (packet delay) }\end{array}$ & Jitter & $\begin{array}{l}\text { Quality } \\
\text { Score }\end{array}$ \\
\hline $6 / 14 / 202021: 55: 0$ & 102.89 .2 .166 & $2.40 \mathrm{Mbps}$ & $0.15 \mathrm{Mbps}$ & $255 \mathrm{~ms}$ & $28 \mathrm{~ms}$ & Good \\
\hline $6 / 14 / 202022: 13: 42$ & 102.89 .2 .166 & $2.14 \mathrm{Mbps}$ & $0.23 \mathrm{Mbps}$ & $227 \mathrm{~ms}$ & $238 \mathrm{~ms}$ & Good \\
\hline $6 / 14 / 202022: 17: 47$ & 102.89 .2 .166 & $0.75 \mathrm{Mbps}$ & $0.28 \mathrm{Mbps}$ & $228 \mathrm{~ms}$ & $5 \mathrm{~ms}$ & Fair \\
\hline $6 / 14 / 202022: 24: 37$ & 102.89 .2 .166 & $4.07 \mathrm{Mbps}$ & $0.41 \mathrm{Mbps}$ & $226 \mathrm{~ms}$ & $6 \mathrm{~ms}$ & Poor \\
\hline $6 / 14 / 202022: 31: 11$ & 102.89 .2 .166 & $2.44 \mathrm{Mbps}$ & $0.44 \mathrm{Mbps}$ & $220 \mathrm{~ms}$ & $9 \mathrm{~ms}$ & Good \\
\hline $6 / 14 / 202022: 44: 40$ & 102.89 .2 .166 & $4.70 \mathrm{Mbps}$ & $0.52 \mathrm{Mbps}$ & $224 \mathrm{~ms}$ & $6 \mathrm{~ms}$ & Good \\
\hline $6 / 14 / 202022: 50: 38$ & 102.89 .2 .166 & $2.11 \mathrm{Mbps}$ & $0.85 \mathrm{Mbps}$ & $232 \mathrm{~ms}$ & $67 \mathrm{~ms}$ & Good \\
\hline $6 / 14 / 202022: 51: 48$ & 102.89 .2 .166 & $5.20 \mathrm{Mbps}$ & $0.56 \mathrm{Mbps}$ & $234 \mathrm{~ms}$ & $6 \mathrm{~ms}$ & Good \\
\hline $6 / 14 / 202022: 53: 48$ & 102.89 .2 .166 & $3.55 \mathrm{Mbps}$ & $0.83 \mathrm{Mbps}$ & $221 \mathrm{~ms}$ & $10 \mathrm{~ms}$ & Bad \\
\hline $6 / 13 / 202022: 59: 33$ & 102.89 .2 .166 & $4.68 \mathrm{Mbps}$ & $1.23 \mathrm{Mbps}$ & $230 \mathrm{~ms}$ & $5 \mathrm{~ms}$ & Fair \\
\hline
\end{tabular}




\begin{tabular}{|c|c|c|c|c|c|c|}
\hline $6 / 13 / 20209: 44: 40$ & 102.89 .2 .166 & $4.70 \mathrm{Mbps}$ & $0.52 \mathrm{Mbps}$ & $224 \mathrm{~ms}$ & $6 \mathrm{~ms}$ & Good \\
\hline $6 / 13 / 20208: 50: 38$ & 102.89 .2 .166 & $2.11 \mathrm{Mbps}$ & $0.85 \mathrm{Mbps}$ & $232 \mathrm{~ms}$ & $7 \mathrm{~ms}$ & Fair \\
\hline $6 / 13 / 20209: 53: 48$ & 102.89 .2 .166 & $3.55 \mathrm{Mbps}$ & $0.83 \mathrm{Mbps}$ & $221 \mathrm{~ms}$ & $10 \mathrm{~ms}$ & Good \\
\hline $6 / 13 / 202022: 51: 33$ & 102.89 .2 .166 & $4.68 \mathrm{Mbps}$ & $1.23 \mathrm{Mbps}$ & $230 \mathrm{~ms}$ & $5 \mathrm{~ms}$ & Good \\
\hline 6/13/2020 12:44:40 & 102.89 .2 .166 & $4.70 \mathrm{Mbps}$ & $0.52 \mathrm{Mbps}$ & $224 \mathrm{~ms}$ & $6 \mathrm{~ms}$ & Poor \\
\hline $6 / 13 / 20201: 50: 38$ & 102.89 .2 .166 & $2.11 \mathrm{Mbps}$ & $0.85 \mathrm{Mbps}$ & $232 \mathrm{~ms}$ & $67 \mathrm{~ms}$ & Good \\
\hline $6 / 13 / 202022: 51: 48$ & 102.89 .2 .166 & $5.20 \mathrm{Mbps}$ & $0.34 \mathrm{Mbps}$ & $234 \mathrm{~ms}$ & $6 \mathrm{~ms}$ & $\mathrm{Bad}$ \\
\hline "6/13/2020 2:23:48" & 102.89 .2 .166 & $3.55 \mathrm{Mbps}$ & $0.83 \mathrm{Mbps}$ & $221 \mathrm{~ms}$ & $10 \mathrm{~ms}$ & Good \\
\hline $6 / 13 / 20202: 12: 33$ & 102.89 .2 .166 & $4.68 \mathrm{Mbps}$ & $1.23 \mathrm{Mbps}$ & $230 \mathrm{~ms}$ & $5 \mathrm{~ms}$ & Fair \\
\hline $6 / 12 / 20205: 20: 40$ & 102.89 .2 .166 & $4.70 \mathrm{Mbps}$ & $0.52 \mathrm{Mbps}$ & $224 \mathrm{~ms}$ & $6 \mathrm{~ms}$ & Good \\
\hline $6 / 12 / 20205: 50: 38$ & 102.89 .2 .166 & $2.11 \mathrm{Mbps}$ & $0.85 \mathrm{Mbps}$ & $232 \mathrm{~ms}$ & $67 \mathrm{~ms}$ & Fair \\
\hline $6 / 12 / 20205: 51: 48$ & 102.89 .2 .166 & $6.20 \mathrm{Mbps}$ & $0.21 \mathrm{Mbps}$ & $234 \mathrm{~ms}$ & $6 \mathrm{~ms}$ & Good \\
\hline $6 / 12 / 2020$ 5:53:48 & 102.89 .2 .166 & $3.55 \mathrm{Mbps}$ & $0.83 \mathrm{Mbps}$ & $221 \mathrm{~ms}$ & $10 \mathrm{~ms}$ & Good \\
\hline $6 / 12 / 202022: 59: 33$ & 102.89.2.166 & $4.68 \mathrm{Mbps}$ & $1.23 \mathrm{Mbps}$ & $230 \mathrm{~ms}$ & $5 \mathrm{~ms}$ & Poor \\
\hline $6 / 12 / 202022: 44: 40$ & 102.89 .2 .166 & $4.70 \mathrm{Mbps}$ & $0.52 \mathrm{Mbps}$ & $224 \mathrm{~ms}$ & $6 \mathrm{~ms}$ & Good \\
\hline $6 / 12 / 202022: 50: 38$ & 102.89 .2 .166 & $2.11 \mathrm{Mbps}$ & $0.85 \mathrm{Mbps}$ & $232 \mathrm{~ms}$ & $67 \mathrm{~ms}$ & Poor \\
\hline 6/12/2020 22:51:48 & 102.89 .2 .166 & $5.20 \mathrm{Mbps}$ & $0.67 \mathrm{Mbps}$ & $234 \mathrm{~ms}$ & $6 \mathrm{~ms}$ & Good \\
\hline $6 / 12 / 202022: 53: 06$ & 102.89 .2 .166 & $3.55 \mathrm{Mbps}$ & $0.83 \mathrm{Mbps}$ & $221 \mathrm{~ms}$ & $10 \mathrm{~ms}$ & Good \\
\hline $6 / 12 / 202022: 59: 33$ & 102.89 .2 & $4.68 \mathrm{Mbps}$ & $1.23 \mathrm{Mbps}$ & $230 \mathrm{~ms}$ & $5 \mathrm{~ms}$ & Good \\
\hline $6 / 11 / 2020$ 22:44:40 & 102.89 .2 .166 & 4.70 Mbps & $0.52 \mathrm{Mbps}$ & $224 \mathrm{~ms}$ & $6 \mathrm{~ms}$ & Good \\
\hline $6 / 11 / 202022: 50: 38$ & 102.89 .2 .166 & $2.11 \mathrm{Mbps}$ & $0.85 \mathrm{Mbps}$ & $232 \mathrm{~ms}$ & $67 \mathrm{~ms}$ & $\mathrm{Bad}$ \\
\hline $6 / 11 / 2020$ 22:51:48 & 102.89 .2 .166 & $5.20 \mathrm{Mbps}$ & $0.41 \mathrm{Mbps}$ & $234 \mathrm{~ms}$ & $6 \mathrm{~ms}$ & Bad \\
\hline $6 / 11 / 202022: 53: 48$ & 102.89 .2 .166 & $3.55 \mathrm{Mbps}$ & $0.83 \mathrm{Mbps}$ & $221 \mathrm{~ms}$ & $10 \mathrm{~ms}$ & Good \\
\hline $6 / 11 / 202022: 59: 33$ & 102.89 .2 .166 & $4.68 \mathrm{Mbps}$ & $1.23 \mathrm{Mbps}$ & $230 \mathrm{~ms}$ & $5 \mathrm{~ms}$ & $\mathrm{Bad}$ \\
\hline 6/11/2020 22:44:40 & 102.89 .2 .166 & 4.70 Mbps & $0.52 \mathrm{Mbps}$ & $224 \mathrm{~ms}$ & $6 \mathrm{~ms}$ & $\mathrm{Bad}$ \\
\hline $6 / 11 / 202022: 50: 38$ & 102.89 .2 .166 & $2.11 \mathrm{Mbps}$ & $0.85 \mathrm{Mbps}$ & $232 \mathrm{~ms}$ & $67 \mathrm{~ms}$ & Good \\
\hline $6 / 11 / 202022: 51: 48$ & 102.89 .2 .166 & $5.20 \mathrm{Mbps}$ & $0.59 \mathrm{Mbps}$ & $234 \mathrm{~ms}$ & $6 \mathrm{~ms}$ & Good \\
\hline $6 / 11 / 202022: 53: 48$ & 102.89 .2 .166 & $3.55 \mathrm{Mbps}$ & $0.83 \mathrm{Mbps}$ & $211 \mathrm{~ms}$ & $10 \mathrm{~ms}$ & Good \\
\hline $6 / 11 / 202022: 59: 73$ & 102.89 .2 .166 & $4.68 \mathrm{Mbps}$ & $1.23 \mathrm{Mbps}$ & $230 \mathrm{~ms}$ & $5 \mathrm{~ms}$ & Fair \\
\hline $6 / 10 / 20204: 44: 40$ & 102.89 .2 .166 & $4.70 \mathrm{Mbps}$ & $0.52 \mathrm{Mbps}$ & $224 \mathrm{~ms}$ & $6 \mathrm{~ms}$ & Good \\
\hline $6 / 10 / 202022: 50: 38$ & 102.89 .2 .166 & $2.11 \mathrm{Mbps}$ & $0.25 \mathrm{Mbps}$ & $232 \mathrm{~ms}$ & $67 \mathrm{~ms}$ & Poor \\
\hline $6 / 10 / 2020$ 22:51:42 & 102.89 .2 .166 & $2.20 \mathrm{Mbps}$ & $0.40 \mathrm{Mbps}$ & $234 \mathrm{~ms}$ & $6 \mathrm{~ms}$ & Poor \\
\hline $6 / 10 / 2020$ 5:53:08 & 102.89 .2 .166 & $3.55 \mathrm{Mbps}$ & $0.83 \mathrm{Mbps}$ & $125 \mathrm{~ms}$ & $10 \mathrm{~ms}$ & Good \\
\hline $6 / 10 / 20207: 59: 33$ & 102.89 .2 .166 & 4.68 Mbps & $1.23 \mathrm{Mbps}$ & $230 \mathrm{~ms}$ & $5 \mathrm{~ms}$ & Good \\
\hline 6/10/2020 9:44:04 & 102.89 .2 .166 & 4.70 Mbps & $0.52 \mathrm{Mbps}$ & $314 \mathrm{~ms}$ & $6 \mathrm{~ms}$ & Fair \\
\hline $6 / 10 / 202022: 50: 38$ & 102.89 .2 .166 & $2.11 \mathrm{Mbps}$ & $0.85 \mathrm{Mbps}$ & $232 \mathrm{~ms}$ & $67 \mathrm{~ms}$ & Good \\
\hline $6 / 10 / 202022: 51: 48$ & 102.89 .2 .166 & $5.20 \mathrm{Mbps}$ & $0.32 \mathrm{Mbps}$ & $234 \mathrm{~ms}$ & $6 \mathrm{~ms}$ & Good \\
\hline $6 / 10 / 2020$ 8:38:48 & 102.89 .2 .166 & $3.55 \mathrm{Mbps}$ & $0.83 \mathrm{Mbps}$ & $221 \mathrm{~ms}$ & $10 \mathrm{~ms}$ & Good \\
\hline $6 / 10 / 20207: 59: 33$ & 102.89 .2 .166 & $4.68 \mathrm{Mbps}$ & $1.23 \mathrm{Mbps}$ & $230 \mathrm{~ms}$ & $5 \mathrm{~ms}$ & Good \\
\hline $6 / 10 / 20207: 30: 40$ & 102.89 .2 .166 & $4.70 \mathrm{Mbps}$ & $0.42 \mathrm{Mbps}$ & $224 \mathrm{~ms}$ & $6 \mathrm{~ms}$ & Fair \\
\hline
\end{tabular}

Table 1 shows the download speed and the upload speed, with the download speed greater than that of the upload speed, reason being that for many users, uploading files is quite a bit slower than downloading files. This is usually normal, because most high-speed Internet connections, including cable modems and DSL, are asymmetric such that, they are designed to provide much better speed for downloading than uploading.
Since most users spend much more time downloading (which includes viewing web pages or multimedia files) than they do uploading, high speed Internet providers have designed their systems to give priority to downloading. So, if your upload speed appears to be slower than your download speed, this is probably expected figure 2 depicts our download and upload speed. 


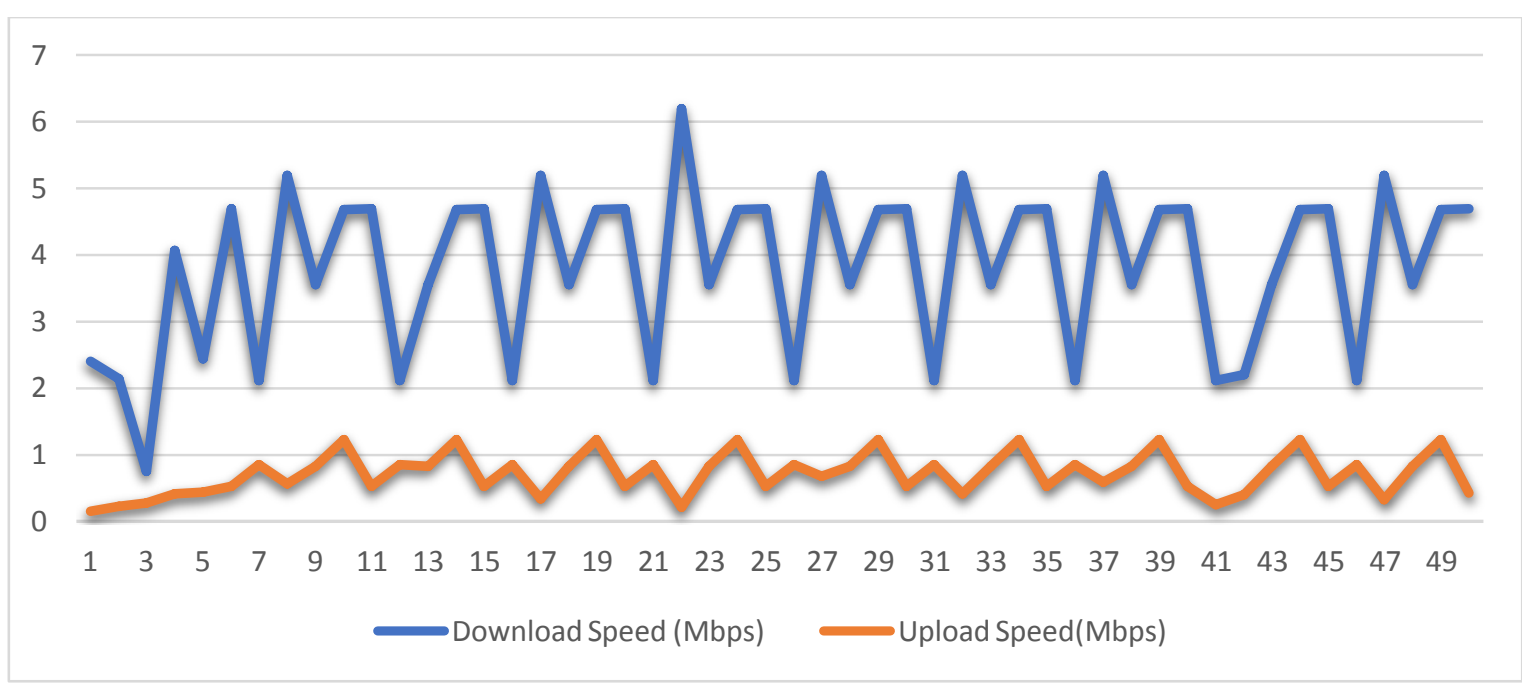

Fig .2: Download vs Upload speed

Also, table 1 shows latency and jitter has it contribute to the overall performance the of network, packet delay and jitter are naturally tied to each other, but they are not the same. Packet Delay is a significant metric in networking that is made up of four key components: processing delay, queueing delay, transmission delay, and propagation delay. It impacts the user experience, and can change based on several factors. Jitter is based off of the delay -

specifically, delay inconsistencies. Jitter is the discrepancy between the delay of two packets. It often results in packet loss and network congestion, which is depicted in Figure 3.

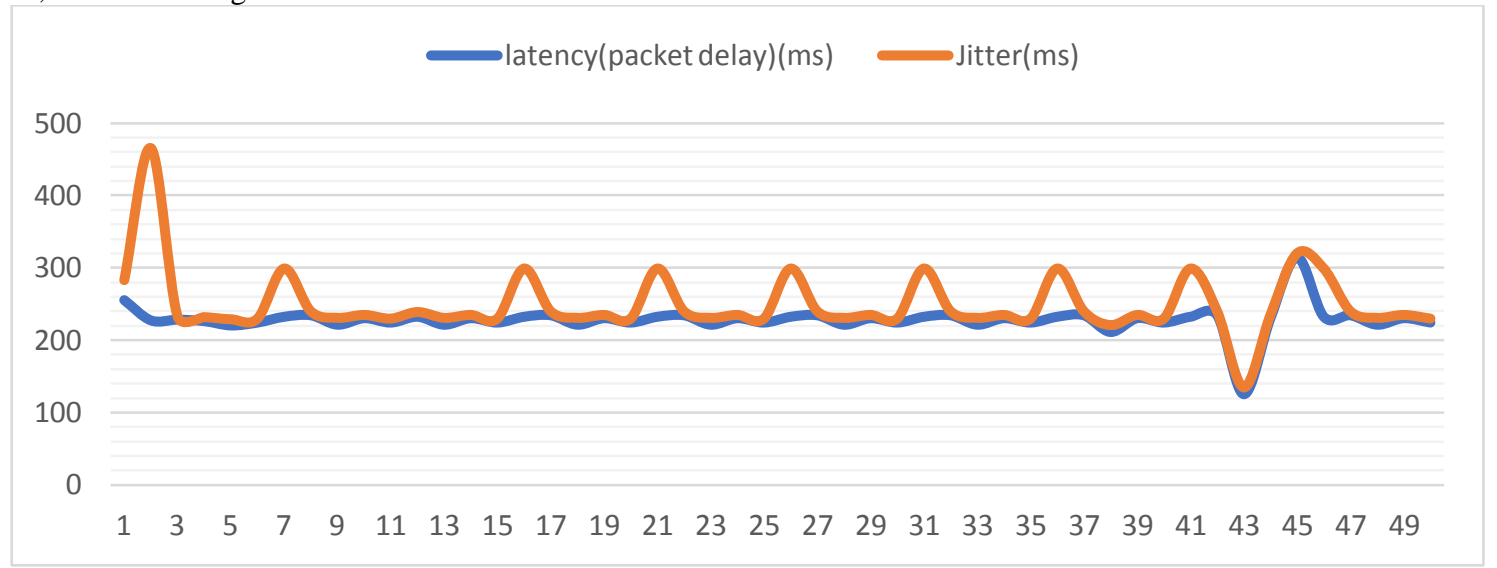

Fig 3 Latency vs jitter

\subsection{Rule Base}

Rules describes the relationship between the input and output linguistic variable which is constructed by their linguistic term.

The general form of a fuzzy rule is defined as a conditional statement. The fuzzy rules are defined using the standard form in the equation below.

$$
R^{l}: I F x_{1} \text { is } \widetilde{F}_{1}^{l} \text { and } \ldots x_{p} \text { is } \widetilde{F}_{p}^{l} \text { THEN } y \text { is } \widetilde{G}_{1}^{l}
$$

Where $1=1, \ldots, N$

We have three (3) input variables and each of these variables have three (3) sets each. From combinational logic, we understand that a truth table of $N$ inputs contain $2^{N}$ rows, one for each possible value of the inputs. From the 4 input variables, the maximum possible number of rules to be used in defining our rule base is given as $3^{3}=81$. As stated earlier, the rule is a collection of $I F-T H E N$ statements.

IF - THEN rules are shown in the table 4.1.

Table 2: Rule Base

\begin{tabular}{|c|l|l|l|l|}
\hline Rule No. & PL & JI & PD & ONP \\
\hline 1. & LOW & LOW & LOW & GOOD \\
\hline 2. & LOW & LOW & MID & GOOD \\
\hline 3. & LOW & LOW & HIGH & GOOD \\
\hline 4. & LOW & MID & LOW & GOOD \\
\hline 5. & LOW & MID & MID & GOOD \\
\hline 6. & LOW & MID & HIGH & AVERAGE \\
\hline 7. & LOW & HIGH & LOW & AVERAGE \\
\hline
\end{tabular}




\begin{tabular}{|l|l|l|l|l|}
\hline 8. & LOW & HIGH & MID & AVERAGE \\
\hline 9. & LOW & HIGH & HIGH & BAD \\
\hline 10. & MID & LOW & LOW & GOOD \\
\hline 11. & MID & LOW & MID & AVERAGE \\
\hline 12. & MID & LOW & HIGH & BAD \\
\hline 13. & MID & MID & LOW & AVERAGE \\
\hline 14. & MID & MID & MID & AVERAGE \\
\hline 15. & MID & MID & HIGH & AVERAGE \\
\hline 16. & MID & HIGH & LOW & AVERAGE \\
\hline 17. & MID & HIGH & MID & BAD \\
\hline 18. & MID & HIGH & HIGH & BAD \\
\hline 19. & HIGH & LOW & LOW & BAD \\
\hline 20. & HIGH & LOW & MID & BAD \\
\hline 21. & HIGH & LOW & HIGH & BAD \\
\hline 22. & HIGH & MID & LOW & AVERAGE \\
\hline 23. & HIGH & MID & MID & GOOD \\
\hline 24. & HIGH & MID & HIGH & BAD \\
\hline 25. & HIGH & HIGH & LOW & AVERAGE \\
\hline 26. & HIGH & HIGH & MID & BAD \\
\hline 27. & HIGH & HIGH & HIGH & BAD \\
\hline
\end{tabular}

To implement the rules in the table above, we used the MATLAB Type-1 Fuzzy Logic Toolbox to achieve all the steps needed to actualize this project implementation, which is shown in Figure 4.

\subsection{Model Formulation}

Network performance shows the measures of service quality of a network as seen by the customer. There are many different ways to measure the performance of a network, as each network is different in nature and design. Basically, performance can be modeled and simulated instead of measured; one example of this is using state transition diagrams to model queuing performance or to use a Network Simulator. The measure of network performance is based on parameters like delay, jitter, packet loss, throughput and many others, depending on the application and measurement scheme. In this research work, the general performance parameters mostly considered in wireless networks are examined.

These include; Packet Delay (PD), Jitter (JI) and Packet Loss

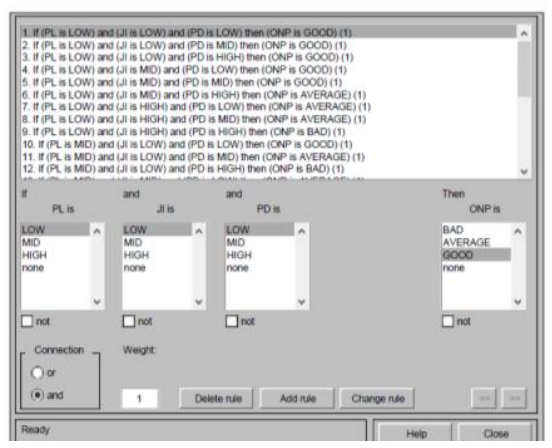

Fig 4: Fuzzy Rule Editor

(PL). These will serve as input variables for fuzzification process of the Fuzzy Logic System. Packet Delay (DE) refers to the amount of time required for

packets to arrive at specified destination measured in milliseconds. Jitter (JI) refers is the undesired deviation from true periodicity of an assumed periodic signal in electronics and telecommunications, often in relation to a reference clock source, which causes loss of synchronization between packets sent, measured in milliseconds. Packet Loss (PL) refers to the percentage of packets lost during transmission.

\subsubsection{Fuzzy Logic System}

We have adopted a T1 FLS based on [8] shown in. Figure 5 Type-1 Fuzzy model. 


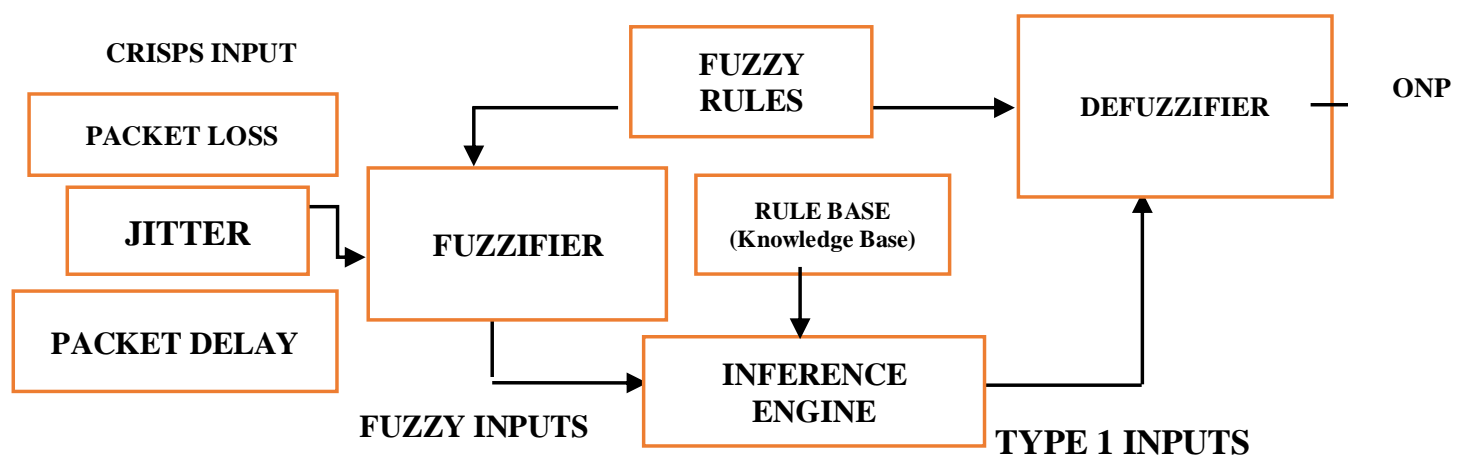

Fig 5: Type 1 Fuzzy Model

The components that makes up the interval type- 1 Model used in this work are discussed as follows:

1. Type 1 Fuzzifier Module: this module maps the crisp input (for determining Optimized Network performance) to the inference engine with triangular membership function so that rules can infer from the knowledge based.

2. Inference Engine: this module evaluates the rules in a rule base gotten from Fuzzification.

3. Defuzzification Module: The output of Interval Type1 Fuzzy Set Mamdani is computed by the composition of membership output and non-membership output functions.

4. Knowledge Base: This is a database of rules (rules are generated from expert's knowledge) to be used by the inference engine.
5. Membership Function: This is a mathematical equation that helps the fuzzification module convert crisp input into fuzzy set.

\subsubsection{Design Architecture}

The conceptual system architecture used for this work is based on the Fuzzy Inference System that basically consist of the MATLAB Graphical User Interface that provides the platform for defining the Fuzzy Rules and Membership Function. The conceptual architecture illustrated below consists of the Knowledge Engine, Knowledge Base which is made of the database model and the Fuzzy Logic Model and the user Interface. The knowledge engine consists of structured and unstructured data, but in this work structured data (Packet Delay (PD), Jitter (JI) and Packet Loss (PL).) are employed in the design of the system. Figure 6 illustrates the conceptual architecture used in this work.

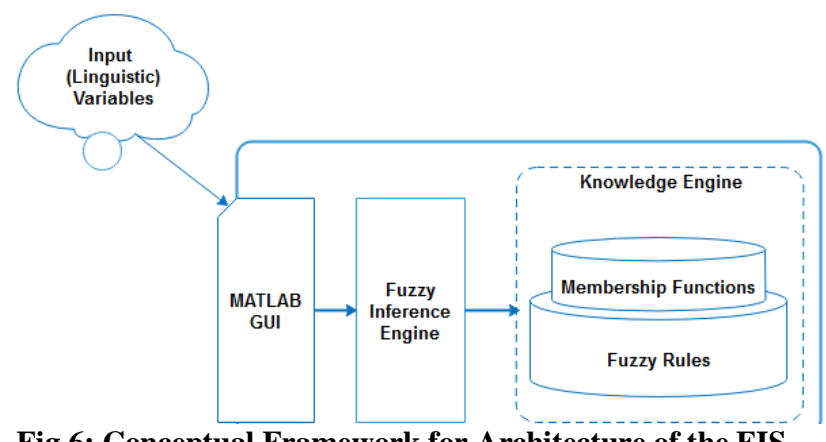

Fig 6: Conceptual Framework for Architecture of the FIS

\subsubsection{System Design Steps}

The Following are the steps used to design the fuzzy Logic of our proposed System.

i. Assign Linguistic Labels PL, JI, PD, and OHD variables.

ii. Define rules for the rule base and obtain a set of firing rules for each range of inputs based on the linguistic labels and membership functions of input variables.

iii. Convert the membership functions into Set for the rules that fired using the equations above.

iv. Obtain the non-zero minimum of the fired rules with their consequence from the composed rules.

v. Perform inference mechanism using Mamdani's method on the linguistic labels.

vi. Composition of the membership and nonmembership with the results (of step 5) to obtain the

$$
\text { crisp input. }
$$

\subsubsection{Fuzzification}

The fuzzification process is carried out on the values of the selected input variables using the membership functions to determine their degree of membership. This converts the crisp quantities into to fuzzy values. This is then used to map the output value specified in the individual rules to an intermediate output measuring fuzzy sets.

The fuzzy linguistics variables and terms for each input parameter are defined as follows;

$$
\begin{array}{cl}
\text { i. } & \text { Packet loss [Low, Mid, and High] } \\
\text { ii. } & \text { Jitter [Low, Average, and High] } \\
\text { iii. } & \text { Packet Delay [Low, Average, and High] }
\end{array}
$$

The OPN output represents level of customer's satisfaction with service quality. The output fuzzy linguistic variable and its terms are 
defined as Optimized Network Performance [Good, Average, Bad]

The universe of discourse for the input and output parameters are as follows;
i. packet loss $[0,100]$ percent
ii. Packet delay $[0,300] \mathrm{ms}$,
iii. Jitter $[0,50] \mathrm{ms}$,
iv. Optimized Network Performance [0, 100], respectively.

The crisp input and output values are converted to fuzzy values by the input and output MFs respectively. A Triangular membership functions (MFs) is used for the evaluation. A Triangular MF curve depends on three parameters b1; b2, and b3, depicted below;

$$
\begin{aligned}
& \mu(x) \\
& =\left\{\begin{array}{cc}
0(x-b 1) /(b 2-b 1) & \text { if } x<1 \leq x<b 2 \\
0(b 3-x) /(b 3-b 2) & \text { if } b 2 \leq x<b 3
\end{array}\right. \\
& \text { if } x>b 3 \text {, }
\end{aligned}
$$

where b2 defines the triangular peak location, while b1 and b3 defines the triangular end points.

\subsubsection{Membership Function Definition}

As earlier established in the previous subsection, we employ the triangular membership function above. Individual range of inputs and output variables is outlined to relate with a fuzzy set that has the same name as the range. We have identified three Linguistic input variables and defined three fuzzy sets for these input variables as well as three fuzzy sets for the output variables. The tables below present a summary of the Linguistic Universe of Discourse.

Triangular membership function in this research are presented below;

\section{i. Packet Loss}

$$
\begin{aligned}
& P L(x)= \\
& \left\{\begin{array}{c}
\text { if } 0<x \leq 10, \quad \text { "LOW" } \\
\text { if } 10<x \leq 25, \quad \text { "MID" } \\
\text { if } 25<x \leq 100,
\end{array}\right.
\end{aligned}
$$

ii.

$$
J I(x)=\left\{\begin{array}{lc}
\text { if } 0<x \leq 15, & \text { "LOW" } \\
\text { if } 15<x \leq 30, & \text { "MID" } \\
\text { if } 30<x \leq 50, & \text { "HIGH" }
\end{array}\right.
$$

iii.

$$
J I(x)=\left\{\begin{array}{cc}
\text { if } 0<x \leq 100, & \text { "LOW" } \\
\text { if } 100<x \leq 200, & \text { "MID" } \\
\text { if } 200<x \leq 300, & \text { "HIGH" }
\end{array}\right.
$$

iv.

$$
\begin{aligned}
& Q N P(x) \\
& =\left\{\begin{array}{c}
\text { if } 0<x \leq 30, \quad " B A D " \\
\text { if } 30<x \leq 70, \\
\text { if } 70<x \leq 300, \quad \text { "GOORAGE" }
\end{array}\right.
\end{aligned}
$$

\subsubsection{Fuzzy Rules}

The fuzzy rules are defined based on observation from data collected from a service provider and from expert experience. An expert, in this case, is the network, the following membership plots shows how the rules are applied in constructing the different membership plots.

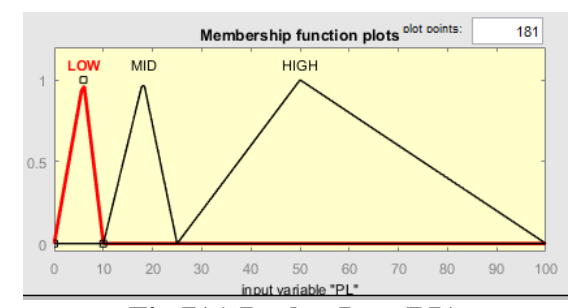

Fig 7(a) Packet Loss(PL)

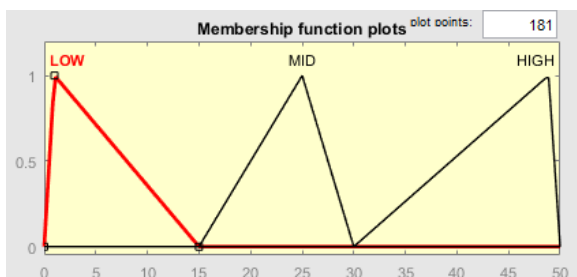

Fig 7(b) Jitter (JI)

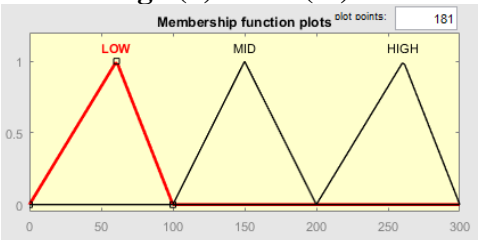

Fig 7(c) Packet Delay(PD)

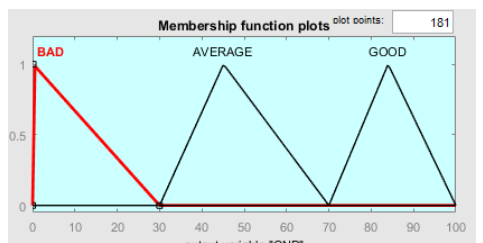

Fig 7(d) Optimized Network Performance(ONP)

Fig. 7a, 7b, 7c, and 7d shows Inputs and output triangular MF plots for i) Packet Loss (PL), (ii) jitter (JI), iii) Packet Delay (PL) and (iv) Optimized Network Performance (ONP).

\subsubsection{Fuzzy Inference Engine}

The Fuzzy inference draws conclusions from existing data. The fuzzy inputs are evaluated to determine the degree to which they belong to each of the appropriate fuzzy sets via membership functions. Aggregation operation method is used to calculate the degree of firing strength, $\beta_{i} \mathrm{i}$ of the condition of rule $\mathrm{i}$ and is given below;

$\beta_{i}=\mathrm{PL}_{i 1}(\mathrm{x} 0) \cap \mathrm{JI}_{i 1}(\mathrm{y} 0) \cap \mathrm{PD}_{i 1}(\mathrm{z} 0)$,

$\mathrm{PL}_{i 2}(\mathrm{x} 0) \cap \quad \mathrm{JI}_{i 2}(\mathrm{y} 0) \cap \quad \mathrm{PD}_{i 2}(\mathrm{z} 0), \quad \mathrm{PL}_{i 3}(\mathrm{x} 0) \cap \quad \mathrm{JI}_{i 3}(\mathrm{y} 0) \cap$

$\mathrm{PD}_{i 3}(\mathrm{z} 0), \ldots, \mathrm{PL}_{\mathrm{im}}(\mathrm{x} 0) \cap \mathrm{JI}_{\mathrm{im}}(\mathrm{y} 0) \cap \mathrm{PD}_{\mathrm{im}}(\mathrm{z} 0)$,

Where $\beta_{i}$ is the matching degree of a given input which satisfies the condition of the ith rule and $i=1,2, \ldots 27$. A rule, say rule 1, will generate a fuzzy membership value PL1, JI1, and PD1 coming from Packet Loss, jitter and packet Delay quantities. A Fuzzy logical AND is applied on the combination of PL1, JI1, and PD1 to evaluate the composite firing strength of the rule. The rules use the input membership values as weighting factors to determine their influence on the fuzzy output sets of the final output (QNP) or conclusion. The degrees of truths $(\mathrm{R})$ of the rules for each rule is

determined by evaluating the non-zero minimum values using the AND operator. Only the rules that get strength higher than 
0 , would fire the output. The root sum square (RSS) inference engine method is used to combine the effects of all applicable rules, scale the functions at their respective magnitudes, and compute the "fuzzy" centroid of the composite area. RSS technique, though more complicated mathematically than other methods, but is choose for this work because it is believed in presenting

the best-weighted influence to all firing rules [13] example

$$
R S S=\left(R_{1}^{2}+R_{2}^{2}+\cdots+R_{n}^{2}\right)^{\frac{1}{2}}=\sqrt{R_{i}^{2}}
$$

\subsubsection{Defuzzification}

The defuzzification is the converse process of fuzzification. The defuzzification process involves converting a fuzzy set or input into a crisp value. The most regularly used defuzzification methods are: maximum, centroid of gravity (COG) and the middle of maxima (MOM). The discrete center of gravity otherwise called Centroid of area defuzzification method is adopted in this research work. Here, each rule is weighted using its normalized weighting factor and the output of the FIS is computed by the summation of all rule outputs. Example

$$
\begin{aligned}
& \text { The Crisp output } \\
& Z Z=\sum_{i}^{27} \boldsymbol{Y}^{\prime}{ }_{K} \boldsymbol{Z}_{\boldsymbol{K}}=\frac{\sum_{i}^{27} \boldsymbol{Y}_{\boldsymbol{K}} \boldsymbol{Z}_{\boldsymbol{K}}}{\sum_{\boldsymbol{i}}^{27} \boldsymbol{Y}_{\boldsymbol{K}}},
\end{aligned}
$$

Where $Y^{\prime}{ }_{K}$ is running is point in a discrete universe, and $\sum_{i}^{27} Y_{K} Z_{K}$ is its membership value in the membership function. The expression can be interpreted as the weighted average of the elements in the support set.

\section{EVALUATION AND DISCUSSION OF RESULTS}

Result Evaluation is an organized assessment of an on-going or completed project. This aim at determining the relevance level of achievement of project objectives, development effectiveness, efficiency, and sustainability. This research work adopted a fuzzy logic system that consist of a rule viewer that is used for varying of different input in parameters to determine how the output will varies based on different instigation.

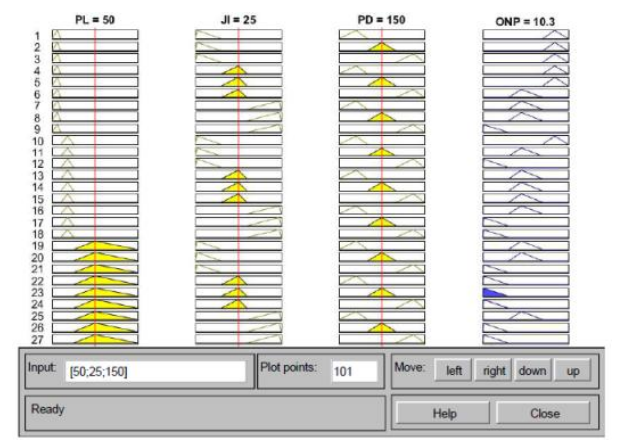

Fig 8: Rule Viewer

From figure 4.4 above, the input variables, PL is set at $50 \%$ which portrays $\mathrm{HGH}$, JI is set at $25 \mathrm{~ms}$ which portrays MID, and PD is set at $150 \mathrm{~ms}$ (milliseconds) which portrays MID and just as the rules define, our output which is the Optimized Network Performance (ONP) is set at 10.3 and according to our defined rules means BAD.

By the output $\mathrm{BAD}$, the system indicates that the high rate of packet loss with medium jitter and long delay during transfer of packets which is mostly cause by high network congestion within a service area causes the next work to perform poorly.
The ONP output variable varies and indicates Network Performance Decisions such "BAD" depending on how the input parameters are varied based on the defined rules.

\subsection{Surface Plots}

Surface plots in fuzzy logic is use depicts 3D representation of each individual variables how it varies in respect to output. Figure. 9 shows a Surface Plot for PL (Packet Loss) against JI (Jitter), the surface plot of Packet Loss against Jitter shows that when there is an medium loss in packets during signal transmission, and jitter which is the vibration in latency that occurs during the time which signal is transmitted to the point which is received the optimized network performance will cause the network to perform averagely, since the congestion rate is low which is the main cause of both packet loss and packet jitter.

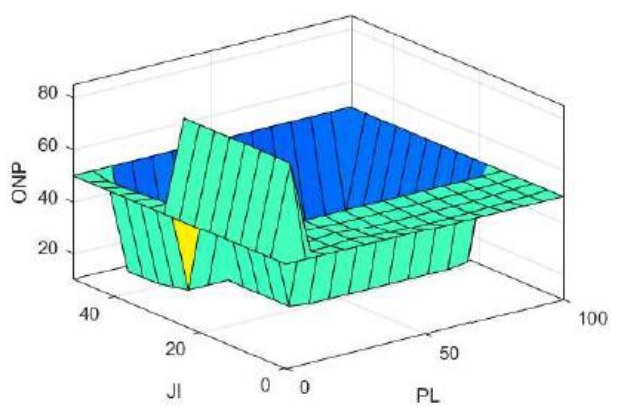

Fig. 9 Surface Plot for PL against JI

Figure 10 shows a Surface Plot for PL (Packet Loss) against PD (Packet Delay), the surface plot of PL against PD shows that when there is a minimal loss in packets, and the delay during transmission from source to destination is minimal too, then the ONP decision factor will initiate an average network performance command since there is low network congestion within the service area.

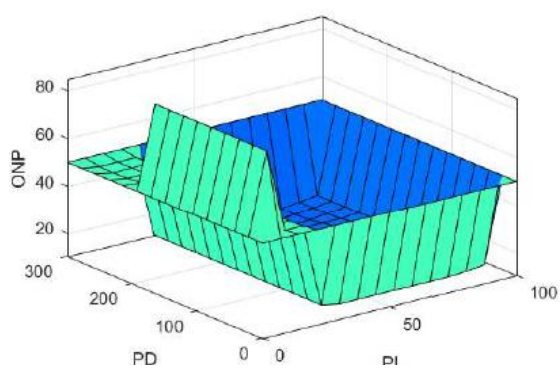

Fig. 10: Surface Plot for PL against PD

Also, Figure. 11 shows a Surface Plot for JI (Jitter) against PD (Packet Delay), the surface plot of JI against PD shows that when there is a minimal delay network coverage area and jitter is low, then the ONP decision factor will initiate a "GOOD" command since there is no congestion in the network, the quality of service will be good and thereby boosting the overall performance of the network. Figure 11 shows Surface Plot for Jitter plotted against Packet Loss 


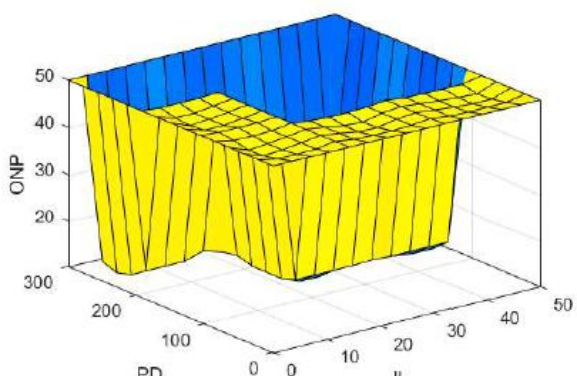

Fig. 11: Surface Plot for JI against PD

Furthermore figure. 12 shows a Surface Plot for PD (Packet Delay) against PL (Packet Loss), the surface plot of PD against PL shows that when there is a low network congestion, then they will be minimal packet loss, also when the delay in delivering packet from source to its destination which collectively makes the network performance to be good.

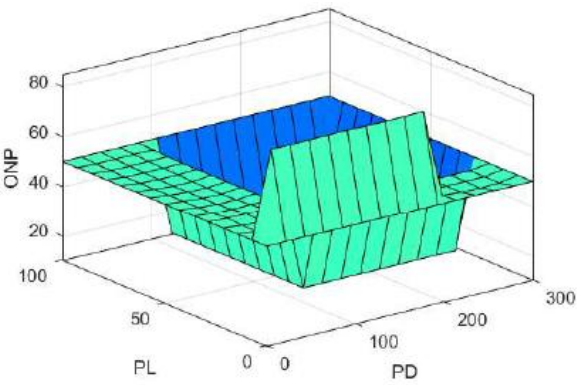

Fig. 12: Surface Plot for $P D$ against $P L$

\subsection{Simulated Inputs Parameters}

We simulate the system using MATLAB Fuzzy Logic tool box functions showing the user interface and fuzzy inference to assist the experimental decision for the best network performance. Figure 12 shows Surface Plot for Packet Delay plotted against Packet Loss. The table 3 indicates inputs used to evaluate Triangular MF as follows:

Table 3: Tested Inputs (Simulated Data)

\begin{tabular}{|c|c|c|c|c|c|}
\hline \multirow[t]{2}{*}{ Conditions } & \multicolumn{3}{|l|}{ Inputs } & \multirow{2}{*}{$\begin{array}{l}\text { Outputs } \\
\text { TRI MF }\end{array}$} & \multirow[t]{2}{*}{ ONP } \\
\hline & $\begin{array}{l}\text { PL (\%) } \\
(0-100)\end{array}$ & $\begin{array}{l}\text { JI (ms) } \\
(0-50)\end{array}$ & $\begin{array}{l}\text { PD (ms) } \\
(0-300)\end{array}$ & & \\
\hline 1. & 50 & 25 & 150 & 10.33333 & BAD \\
\hline 2. & 25 & 40 & 300 & 50 & AVERAGE \\
\hline 3. & 30 & 23 & 50 & 49.52934 & AVERAGE \\
\hline 4. & 17 & 35 & 60 & 50 & AVERAGE \\
\hline 5. & 29 & 50 & 150 & 50 & AVERAGE \\
\hline 6. & 45 & 17 & 250 & 84.72055 & GOOD \\
\hline 7. & 10 & 19 & 280 & 50 & AVERAGE \\
\hline 8. & 7 & 18 & 97 & 84.94276 & GOOD \\
\hline 9. & 100 & 50 & 300 & 50 & AVERAGE \\
\hline 10. & 78 & 10 & 4 & 49.80792 & AVERAGE \\
\hline 11. & 60 & 25 & 200 & 50 & AVERAGE \\
\hline 12. & 4 & 50 & 45 & 50 & AVERAGE \\
\hline 13. & 80 & 12 & 140 & 49.50063 & AVERAGE \\
\hline 14. & 45 & 25 & 45 & 84.70212 & GOOD \\
\hline 15. & 70 & 23 & 12 & 84.77512 & GOOD \\
\hline 16. & 100 & 56 & 45 & 50 & AVERAGE \\
\hline 17. & 34 & 4 & 56 & 49.19221 & AVERAGE \\
\hline 18. & 12 & 12 & 78 & 84.88135 & GOOD \\
\hline 19. & 29 & 23 & 90 & 49.64233 & AVERAGE \\
\hline 20. & 67 & 45 & 130 & 11.4192 & BAD \\
\hline
\end{tabular}

Figure 13 illustrates the simulated input parameters for network performance, which constitutes packet loss, jitter and packet delay. Packet delays shows that when the duration or time in delivering of packets to their destination is high and all other input parameter are averagely or low then the network performance will be Bad, but when the delay is low then it easier to obtained an optimal performance of the network. These three parameters are they parameters that's mostly hinders network performance during congestion which are importance when considering optimizing network performance for Quality-of-Service provisioning.

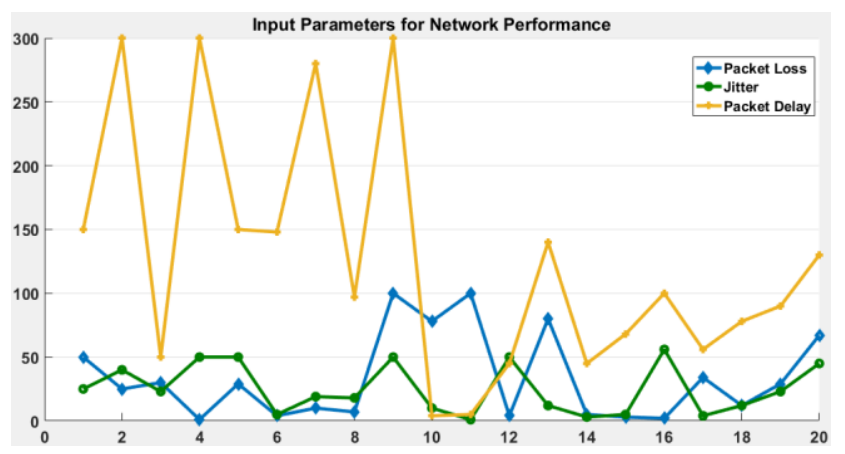

Fig. 13 Network Performance Inputs 
Furthermore, Figure 14 illustrates the simulated network performance output, which constitutes packet loss, jitter and packet delay. These three parameters are combined for the performance of the network which perform better than that's of the existing system that uses queue process in admitting packets to the destination based on queue sized.

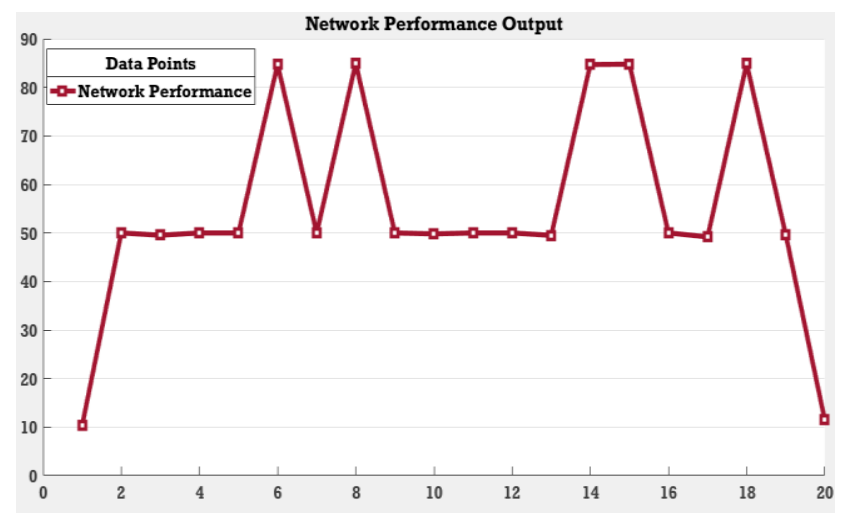

Fig. 14: Network Performance Output

\section{ANALYSIS AND DISCUSSION}

Analysis of the existing system which uses Active Queue Management (AQM) algorithm shows some flaws, in that an effective utilization of bandwidth cannot be achieved and also can drop packets has long as possible due to the queue size, which hinders the performance of the network, which can lead to a poor quality of service (QoS) delivery and low performance in network services. The existing system further shows that AQM (Active Queue Management) algorithm, cannot handle some packet delivery uncertainties.

Nevertheless, the proposed system using Interval Type 1 Fuzzy Logic model proved a better option to the AQM (Active Queue Management) algorithm. Moreover, the Interval type 1 model shows a reduction in handling uncertainties in network performance optimization, hereby improving the efficiency and Quality of Service provisioning of the Wireless Networks.

Today, the demand for better and good quality of service (QoS) in wireless network is one of the most essential

requirements in today's fast paced world of Information Technology (IT). Information has become the highest dealt commodity in our world today. Modern businesses, schools, governments, and even individuals depend largely on daily information that must be accessed in real time, to smoothly access this information they arise a need for good quality of service delivery will in turn enhanced better network performance.

The problems on quality of service (QoS) delivery are more intricate for wireless network access systems than for wire line systems. This is because wireless access networks experience high bit error rate (BER), limited bandwidth, user contention and frequent mobility, radio inference, and diverse traffic characteristics. These wireless access issues have brought about data delivery problems such as slow peripheral access, data errors, dropouts, unnecessary retransmissions, traffic congestion, out of sequence data packets, latency, and jitter, etc. The need for intelligent approach to providing optimization mechanisms that enhance customer service satisfaction and increase revenue for network service providers cannot be overstressed.

Though various Network Performance Optimization algorithm has been proposed progressively, but they have been numerous challenges encounter during optimization of network performance such as, the number of parameters use for network optimization, approaches, employed in optimization process and the ability to handle uncertainties especially when face with a lot of data associated in network access, this challenge can lead to, congestion, that can give rise to poor network performance.

\section{CONCLUSION}

Basically, to meet the demand for Quality of service (QoS) provisioning and good networks performance, the development of an Optimal Networks Performance (ONP) was considered. The Paper study the problem of existing loopholes which may likely adjust the provision of such required Quality of Service (seamless connectivity). Hence, the work presents a typical deployment setup of a WLAN by specifying essential network elements and providing the equivalent available service providers. Again, the work adopted a Computational Intelligence (CI) approach using Type 1 Fuzzy Logic system and subjected the obtained operational field data for simulation Results demonstrates an effective minimization of congestions with the representative networks environments. Research findings further validates efficient network performance. Consequently, the evaluation carried out with Triangular Membership Functions (TMF). demonstrate an optimized WLAN Performance with QOS provisioning within a given service area.

\section{ACKNOWLEDGMENT}

Special Acknowledgement to TETFUND, Nigeria for contributing to Research and Development (R\&D) in Tertiary Institutions in Nigeria.

\section{REFERENCES}

[1] Akram M. and Hatem M. (2017). Computer Network Performance management using a Simple Network Management Protocol, International Journal of Computing Academic Research (IJCAR) ISSN 2305-9184, Volume 6, Number 2, pp.50-58, Egypt.

[2] Akhilesh K. and Ompal S, (2012). 5G Technology Redefining wireless Communication in upcoming years, International Journal of Computer Science and Management Research Vol 1 Issue 1.

[3] Alikira R (2012). Evaluation of WLAN Security and Performance, Munich, GRIN Verlag, https://www.grin.com/document/205389.

[4] Carlos A., Octavio J., Miguel J., (2017). Performance Optimization Model n802.11n Networks Using MultiObjective Programming. Applied Mathematical Sciences, Vol. 11, 2017, no. 59, 2907 - 2918 HIKARI.

[5] Kuteyi A (2015). Network Performance Analysis Within A Local Area network. https://www.academia.edu/14397915/

[6] Kaidioglu, R (2010). Performance benchmarking of cellular network operators in Turkey. Graduate School of Natural and Applied Sciences of ATILIM University Turkey, Electrical and Electronic Engineering.

[7] Lopa, J. (2015). Evolution of Mobile Generation Technology: $1 \mathrm{G}$ to $5 \mathrm{G}$ and Review of Upcoming Wireless Technology 5G. International Journal of Modern Trends in Engineering and Research (IJMTER) Volume 02, Issue 10, ISSN (Online):2349-9745; ISSN (Print):2393-8161.

[8] Mendel, J. M., Hongwei,W. (2007). New results about 6 the centroid of an interval type-2 fuzzy set, including the 
centroid of a fuzzy granule. Information Sciences, 177(2):360-377.

[9] Munam, Ghazanfar, Carsten, Khurram, (2011). Network Performance Optimization: A Case Study of Enterprise Network Simulated in OPNET.

[10]Nandhini, Jagadhesan, Anusuja (2016). An Optimization of Wireless Network Security System, International Journal of Computer Science and Information Technology Research ISSN 2348-120X (online) Vol. 4, Issue 3, pp: (296-301).

[11] Nosiri C, Onyenwe M, Ekwueme U, (2019). Electrical and Electronic Engineering Department, Federal University of Technology, Fuzzy Logic Implementation for Enhanced WCDMA Network Using Selected KPIs Owerri, Nigeria

[12] Richa R, Shrivastava S, and Sarita S, (2016). Performance Analysis of Fuzzy based RED for Congestion Control in MANET International Journal of Smart Home Vol. 10, No. 5, pp. 231-240

[13] Umoh U, Nwachukwu E, and Okure O., (2010). Fuzzy rule-based framework for effective control of profitability in a paper recycling plant, Global J. Computer. Sci.
Technol. pp 56-67.

[14] Imeh J. Umoren, Prince Asagba and Olumide Owolabi (2014). Handover Manageability and Performance Modeling in CDMA Mobile Communication Networks, International Institute for Science, Technology and Education (IISTEE) and Computing Information Systems Development Informatics and Allied research -Journal (CISDA). Vol 5 No. 1, ISSN: 2167 -1710, page 27-42.

[15] Imeh J. Umoren, Daniel E. Asuquo, Onukwugha Gilean, Mfon Esang (2019. Performability of Retransmission of Loss Packets in Wireless Sensor Networks. Comput. Inf. Sci. 12(2): 71-86, Canada.

[16] Imeh J. Umoren and Saviour J. Inyang (2021). Methodical Performance Modelling of Mobile Broadband Networks with Soft Computing Model. International Journal of Computer Applications 174(25):7-21, NY, USA.

[17] Ye, Quanmin (2014). Cross-layer schemes for performance optimization in wireless networks (Doctoral Dissertations. 2328.

[18] Yogesh Misra (2012). A review on application of fuzzy logic in increasing the Efficiency of Industrial process" (IJLTET) Vol. 1 ,11 ISSN: 2278- 621X. 